\title{
Freshman Undergraduate Biology Students' Difficulties with the Concept of Common Ancestry
}

\author{
Brian T. White • Steven Yamamoto
}

Published online: 30 November 2011

(C) Springer Science+Business Media, LLC 2011

\begin{abstract}
All life on earth descended from a single common ancestor that existed several billion years ago; thus, any pair of organisms will have had a common ancestor at some point in their history. This concept is fundamental to an understanding of evolution and phylogeny. Developing an understanding of this concept is an important goal of evolution education and a part of most high school and college biology curricula. This study examines freshman undergraduate biology majors' understanding and application of the concept of common ancestry. We used a survey that asked students to provide a brief definition of common ancestry and to rate their confidence that different pairs of organisms shared a common ancestor. Our results show that, although many students in our sample could give a satisfactory definition of common ancestry, the overwhelming majority failed to apply their definitions correctly when assessing the likelihood that the pairs of organisms shared common ancestors. Instead, we found that these students do not treat common ancestry as a binary (yes/no) trait, but instead see it as a continuum from less probable to more probable. These students are more likely to think that closely related organisms have a common ancestor than those that are more distantly related and that humans are less likely to be connected to common ancestors than nonhuman organisms. This pattern is highly consistent from student to student and has important implications for teaching evolution.
\end{abstract}

Keywords Education · Common ancestry · Evolution misconceptions · Undergraduate students

B. T. White $(\bowtie) \cdot S$. Yamamoto

Biology Department, University of Massachusetts Boston, 100 Morrissey Blvd,

Boston, MA 02125, USA

e-mail: brian.white@umb.edu

\section{Introduction}

The descent of all living things from a single common ancestor is a core component of modern evolutionary theory, as is its corollary, that any pair of organisms, no matter how distantly related, will have had a common ancestor at some point in their recent or distant past (Freeman et al. 2007). As a result, this concept is an important part of the teaching of evolution in K-12 education and a foundation for further study in biology at the college level. The National Science Education Standards (NRC 1996) for grades 5-8 states, "Millions of species of animals, plants, and microorganisms are alive today. Although different species might look dissimilar, the unity among organisms becomes apparent from an analysis of internal structures, the similarity of their chemical processes, and the evidence of common ancestry." For grades 9-12 it states, "The millions of different species of plants, animals, and microorganisms that live on earth today are related by descent from common ancestors." Of the 41 state K-12 science standards reviewed in 2005 (Swanson 2005), 20 included common ancestry. It is also implicit in many other components of the K-12 biology curriculum including, for example, discussions of the common structural, genetic, and biochemical features of living things.

Although the evidence for common ancestry of closely related organisms is often easy to observe, this becomes more challenging when organisms are more distantly related and the homologies less obvious. Darwin (1859) recognized this as he described in The Origin of Species (chapter 14; pp 454-455):

It may be asked how far I extend the doctrine of the modification of species. The question is difficult to answer, because the more distinct the forms are which we may consider, by so much the arguments fall away 
in force...Therefore I cannot doubt that the theory of descent with modification embraces all the members of the same class. I believe that animals have descended from at most only four or five progenitors, and plants from an equal or lesser number.

Analogy would lead me one step further, namely, to the belief that all animals and plants have descended from some one prototype. But analogy may be a deceitful guide...Therefore I should infer from analogy that probably all the organic beings which have ever lived on this earth have descended from some one primordial form, into which life was first breathed.

Given the challenging nature of this concept, it is not surprising that many people hold misconceptions about common ancestry. Previous work has explored these difficulties. In a study where undergraduate students were surveyed about core evolutionary concepts, Nadelson and Southerland (2010) found fewer than $50 \%$ of freshman-level students were able to correctly answer questions addressing common descent. Poling and Evans (2004) surveyed a sample of 132 college-educated adults about their understanding of core evolutionary concepts. Among their questions were eight sets of two or three organisms. Subjects were asked to rate on a 5-level Likert scale the extent to which they agreed that each set of organisms shared a common ancestor. It is important to note that, because all organisms have a common ancestor in their recent or distant past, that the correct answer to every question of the form "Do organisms X and $\mathrm{Y}$ have a common ancestor?" is unequivocally "Yes." In spite of this, most subjects did not think that all groups shared common ancestors. Furthermore, most subjects on average were less likely to agree that species sets with greater taxonomic distance had common ancestors than more closely related species sets. These studies suggest that difficulties with this concept persist in spite of explicit coverage in K-12 education.

Poling and Evans' (2004) study explored the understandings of college-educated adults, both science and nonscience majors; in many cases, many years after college. Our study expands on their work and explores a targeted student population, freshman undergraduate biology students. These students are of particular interest because their understanding of evolutionary concepts, including common ancestry, will serve as a foundation for their college-level work in biology and thus their understanding of evolution as informed citizens, practicing scientists, or health professionals.

We assess freshman biology students' understanding of common ancestry using a survey that is based on that developed by Poling and Evans (2004). Our results show that these students display some of the difficulties with this concept that Darwin (1859) discussed and Poling and Evans (2004) found in college-educated adults. Our survey and study design allows us to explore these difficulties in more detail than Poling and Evans (2004). Our findings have important implications for teaching this core evolutionary concept.

\section{Course Context and Student Population}

This survey was administered to two cohorts of students enrolled in General Biology I at the University of Massachusetts Boston (UMB). The first cohort completed the survey at the end of the fall semester of 2008 and the second cohort completed it at the end of the Fall semester 2009. General Biology I is a typical lecture/lab course for biology majors that covers genetics, cell biology, biochemistry, and molecular biology. It emphasizes the unity of all living organisms at the molecular level. Because the UMB is an urban public university, the student body is highly diverse, with $42 \%$ nonwhite students. Most have graduated from high school in Massachusetts, where common ancestry is a component of the state K-12 science framework (MassDOE 2006). Thus, the students in our study had been exposed, both directly and indirectly, to the idea of common ancestry before taking the survey.

Students' participation in the survey was voluntary. Students received a small amount of course credit for turning in the survey; they were informed that the amount of credit did not depend on whether their answers were correct or not.

\section{Statistical Methods}

Students were asked to respond on a 5-level Likert-style scale $(1=$ strongly disagree, $2=$ disagree, $3=$ not sure, $4=$ agree, $5=$ strongly agree) to statements of the form, " $<$ organism $A>$ and $<$ organism B $>$ have a common ancestor." Thus, a higher response level indicates a higher level of agreement that a set of organisms had a common ancestor. Although this is an ordinal scale, it is not an interval scale, so we chose proportional odds logistic regression (POLR) for our analyses. POLR analysis models the effects of predictor variables on the probabilities of each of the ordinal response levels and assumes that the effect of each of the predictor variables is the same for each of the response levels. POLR analysis produces coefficients of a linear model that predicts the response as well as the significance of each coefficient; these coefficients correspond to the effect on the natural logarithm of the odds ratio of each predictor variable. The odds ratios measure the odds of a response being at a particular level compared to the next lower level. Thus, a positive coefficient indicates that increases in a particular predictor increase the changes of higher-level responses and a negative coefficient indicates that increases in the predictor decrease the chance of high-level responses. When measuring the relationship 
between response and taxonomic distance, we specified the taxonomic distance between any two organisms using the following numerical scale: $1=$ different species, $2=$ different genera, $3=$ different families, $4=$ different orders, $5=$ different classes, $6=$ different phyla, and $7=$ different kingdoms. Measurements of correlation between response level and taxonomic distance for individual students were calculated using Kendall's tau. Principal components analysis was carried out using data that had been scaled to unit variance and centered to a mean of zero via a singular value decomposition of the data matrix (Venables and Ripley 2002). Qualitatively similar results were obtained when we carried out alternative nonparametric tests (Wilcoxon-Mann-Whitney test for binary predictor variables; Jonckheere-Terpestra test for trends) on our data. All calculations were performed using $\mathrm{R}$ (version 2.10.0; www.r-project.org) or Microsoft Excel 2004 (version 11.6).

\section{Results and Discussion}

This article describes the results of two studies, one carried out in 2008 and the other in 2009; the studies each used a different version of the survey. The first was a pilot that provided information that informed the development of the second. The results of this second study will be explored in more depth.

\section{Fall 2008 Pilot Study}

This study used a survey based on the one developed by Poling and Evans (2004). As part of their survey, they asked their subjects to respond on a 5 -level Likert-style scale $(1=$ strongly disagree, $2=$ disagree, $3=$ not sure, $4=$ agree, $5=$ strongly agree) to eight statements similar to and including, "Gorillas, monkeys, and chimpanzees all have a common ancestor." Our survey included all of their questions along with five more that we developed. Two versions of the survey were administered. In version A, questions were presented in decreasing order of diversity (more diverse first; least diverse last); in version $B$, the same questions were asked in reverse order. Surveys were shuffled and handed out to students randomly. It is important to note that this question did not ask if the organisms shared a recent common ancestor; thus, the only correct answer to all of these questions is 5 .

The survey was completed by 272 students in General Biology in lecture near the end of the fall 2008 semester; these students represented $83 \%$ of those enrolled in the course. Although the correct answer is "Strongly Agree" (5) to all 13 questions, only $7 \%$ of the students gave this response. Students' responses were analyzed with a POLR model that included taxonomic distance (1-7) and question order (highto-low and low-to-high); this is summarized in Table 1. The POLR model predicted the response significantly better than
Table 1 Results of POLR analysis of students' responses to versions $\mathrm{A}$ and $\mathrm{B}$

\begin{tabular}{lcc}
\hline Factor & Coefficient & $P$ value \\
\hline One level increase in taxonomic difference & -0.50 & $<10^{-16}$ \\
Question order (changing from low-to-high & 0.21 & $6.26 \times$ \\
to high-to-low) & & $10^{-9}$ \\
\hline
\end{tabular}

The coefficient is the natural logarithm of the change in the odds ratio resulting from a change in the corresponding factor

a null model with no parameters (model likelihood $\chi^{2}<0.0001$ ); it explained roughly $19 \%$ of the variance in responses (Nagelkerke's $\left.R^{2}=0.192\right)$. Analysis of students' responses showed a strong negative correlation between agreement and decreasing distance. This is similar to Poling and Evans' (2004) finding that their subjects were less confident that distantly related organisms shared a common ancestor.

Interestingly, the POLR results show that the order in which the statements were presented had a significant influence on students' agreement level. On average, students who received the survey where the questions began with high diversity organism sets (version A) showed a higher level of agreement with all of the statements than those who received the survey where the questions began with low diversity sets (version B). The significant effect of question order shows that the students' responses are dependent on the context in which individual questions are asked, suggesting that their understanding of common ancestry is not completely fixed. This context effect has been observed with other science misconceptions; for example, in physics where Mildenhall and Williams (2001) found that students' use of Aristotelian or Newtonian concepts of motion was not consistent and, in fact, depended on the magnitudes of the quantities (forces, masses, etc.) involved.

Finally, Poling and Evans (2004) reported statistical analyses of their subjects' responses to their questions. Using these data, we were able to compare the responses of the two study groups to the same eight questions. The General Biology students' average responses showed a higher level of agreement for each question than Poling and Evans' (2004) college-educated adults; for seven of the eight questions, the difference was significant (data not shown). This difference is consistent with Poling and Evans' (2004) finding that subjects' understanding of common ancestry was positively correlated with education level and number of biology courses taken.

\section{Fall 2009 Study}

The results from the pilot study were provocative; however, there were five serious issues with the survey design that made it difficult to draw strong conclusions. Each of these issues was addressed in design of the second survey. 
First, the sets of organisms used in the pilot study were not well chosen to explore the effects of taxonomic distance on response level. Some of the questions included three organisms, while others included only two. Furthermore, while some organisms were described with very specific names (for example, "coyotes"), others questions used less specific terms (for example, "monkeys"). Both of these make assignment of a particular taxonomic distance to a given question challenging for students and investigators. Finally, the different questions were not distributed evenly with respect to taxonomic distance-most involved level 4, 5 , and 6 differences with few extremes. Based on this, all questions in the second survey involved pairs of organisms described in unambiguous terms and well distributed by taxonomic distance. The questions used are shown in Table 2; the number in the designation of each question indicates the taxonomic distance: 1 (different species) through 7 (different kingdoms).

Second, because people often view humans as exceptions in biological evolution (Pinarbasi and Canpolat 2003), it is important to compare responses to similar questions involving both humans and nonhuman organisms. Although the pilot survey did include humans in some questions, these questions did not involve a range of taxonomic distances. The revised version included questions with and without humans at a wide range of taxonomic distances. Questions involving humans are designated with " $\mathrm{H}$ "; those that do not are designated with "N" in Table 1.

Third, although the pilot survey assumed that students understood the meaning of the statement "Organism A and Organism B have a common ancestor," the survey did not include any measure to assess this understanding. Thus, in the fall 2009 study, version C of the survey asked students to "Briefly explain what the statement 'Organism A and Organism B have a common ancestor' means" before the questions listed in Table 2; versions D and E included only the questions in Table 2.

Fourth, the pilot survey asked the students to respond to a yes-or-no question ("Do $\mathrm{X}$ and $\mathrm{Y}$ have a common ancestor?") on a 5-level scale. It is possible that this led the students to think that the question was not a binary choice and, as a result, they felt obliged to give answers that fell on a continuum. In effect, the design of the survey may have encouraged incorrect responses. To explore this, version $\mathrm{E}$ asked students to respond to each statement on a simplified scale with only three levels $(1=$ no, $2=$ not sure, $3=y e s)$. Here again, the only correct answer to all of these questions is 3 .

Finally, because the pilot survey asked 13 questions all having the same correct answer and the questions appeared in order of taxonomic distance, students' responses might have reflected typical multiple-choice test-taking strategy-where the correct answer is never the same for all questions - rather than application of their understanding of common ancestry. To avoid this structural issue, the order of the questions was scrambled with respect to taxonomic distance in all versions of the final survey; the same scrambled order was used in all three versions.

The survey statements are listed in Table 2. Statements are listed in order of taxonomic distance; their order in the survey is indicated by the question number. The differences between the three versions of the survey used in the fall 2009 study are summarized in Table 3.

At the end of the fall 2009 semester of General Biology I, students completed one of the three versions of the survey shown in Table 3; surveys were randomly handed to each student. Of the 381 students in the course, $75.6 \%$ completed a survey. To assess the degree to which the surveys were distributed to representative samples of the class, we calculated the average final grade point average (GPA; $0-4$
Table 2 Final survey statements

Statements are listed in order of increasing taxonomic distance. The number indicates the order of the statements in the survey. The designation is used in the figures: $\mathrm{N} / \mathrm{H}$ indicates non-human or Human, respectively; the first digit indicates the taxonomic distance $(1=$ different species; $7=$ different kingdoms); the last digit, if present, indicates more than one statement at the same level of taxonomic distance

\begin{tabular}{lll}
\hline Question number & Designation & Statement \\
\hline 8 & $\mathrm{~N} 1$ & Dogs and wolves have a common ancestor \\
2 & $\mathrm{~N} 2$ & Pears and apples have a common ancestor \\
13 & $\mathrm{~N} 2.1$ & Rats and mice have a common ancestor \\
9 & $\mathrm{~N} 3$ & Horse flies and fruit flies have a common ancestor \\
4 & $\mathrm{H} 3$ & Baboons and humans have a common ancestor \\
1 & $\mathrm{~N} 4$ & Whales and zebras have a common ancestor \\
7 & $\mathrm{~N} 4.1$ & Rats and dogs have a common ancestor \\
3 & $\mathrm{~N} 5$ & Crocodiles and dolphins have a common ancestor \\
11 & $\mathrm{H} 5$ & Salamanders and humans have a common ancestor \\
5 & $\mathrm{~N} 6$ & Zebras and snails have a common ancestor \\
10 & $\mathrm{~N} 6.1$ & Earthworms and mosquitoes have a common ancestor \\
12 & $\mathrm{~N} 7$ & Tuna fish and pumpkins have a common ancestor \\
6 & $\mathrm{H} 7$ & Cucumbers and humans have a common ancestor \\
\hline
\end{tabular}


Table 3 Survey versions used in study

\begin{tabular}{lll}
\hline Survey version & Response choices & Common ancestor definition \\
\hline C & 5-Level & Yes \\
D & 5-Level & No \\
E & 3-Level & No \\
\hline
\end{tabular}

This shows the three versions of the survey used in the study. Response choices describe the number of levels used in the Likerttype response choices. Common ancestor definition indicates whether students were asked to define what it means for two organisms to have a common ancestor before responding to the statements

scale) for the students receiving each version of the survey. These data are shown in Table 4. While the differences between students receiving versions $\mathrm{C}$ and version $\mathrm{D}$ as well as the difference between version $\mathrm{D}$ and $\mathrm{E}$ were nonsignificant (Wilcoxon-Mann-Whitney test; $p=0.46$ and $p=0.088$ respectively), the difference between version $\mathrm{C}$ and $\mathrm{E}$ was significant (Wilcoxon-Mann-Whitney test; $p=0.006$ ). Thus, while it is appropriate to compare $\mathrm{C}$ to $\mathrm{D}$ and $\mathrm{D}$ to $\mathrm{E}$, comparisons between versions $\mathrm{C}$ and $\mathrm{E}$ are not appropriate as they appear to be non-equivalent samples of the student population.

We began by looking at the fraction of students who answered all of the questions correctly; that is, students who chose "Strongly Agree" or "Yes" to each question. As in the pilot study, by this criterion, very few students (version C, $6.4 \%$; version $\mathrm{D}, 11.3 \%$; version $\mathrm{E}, 5.2 \%$ ) responded to all of the statements correctly.

Next, we scored the students' definitions of common ancestor from those who completed version $\mathrm{C}$ of the survey. Each of the 94 responses was scored by the two investigators independently; the two scorers agreed $86.2 \%$ of the time. Responses were scored as belonging to one of three categories that correspond to how a short answer question like this would likely be graded on an exam:

- Full credit (44\%). These answers specifically mentioned that organism A and B had descended from a single common species that had existed in the past.

- Part credit (41\%). These answers stated that organism A and B were "related" or "had similar traits" without specifically mentioning common descent.

- No credit $(15 \%)$. These answers were left blank or did not answer the question.

Table 4 Student data for each survey version

\begin{tabular}{llc}
\hline Survey version & Number of students & Mean GPA \\
\hline C & 94 & 2.97 \\
D & 97 & 2.73 \\
E & 97 & 2.44 \\
\hline
\end{tabular}

This shows the number of students who completed each version of the survey and their average course GPAs
From this, slightly less than half of the students could give a fully satisfactory definition of common ancestry. Because of the results of the GPA comparison, while it is appropriate to expect that students completing version $\mathrm{D}$ of the survey would show similar frequencies of responses, it is not possible to draw this conclusion about the students who completed version E. This is similar to the findings of Nadelson and Southerland (2010), who found that $50 \%$ or fewer of their introductory biology students correctly answered their survey questions that dealt with common ancestry.

Although the results of the pilot study were consistent with students having interpreted the question as "Organisms A and B have a recent common ancestor," none of the students' definitions of common ancestor included the word 'recent' or emphasized the ancestor being recent. Thus, it is likely that the students interpreted the question as it was asked - whether or not a common ancestor ever existed.

We next analyzed the data from versions $\mathrm{C}$ and $\mathrm{D}$ of the survey to determine the effects, if any, of the predictor variables (taxonomic distance, the inclusion of humans in the question, and being asked to define common ancestor) on students' level of response. Students' responses were analyzed with a POLR model that included these three predictor variables; the POLR model predicted the response significantly better than a null model with no parameters (model likelihood $\left.\chi^{2}<0.0001\right)$ and it explained roughly $30 \%$ of the variance in responses (Nagelkerke's $R^{2}=0.306$ ). A summary of the POLR results are shown in Table 5; a graph of the data from students who completed version $\mathrm{C}$ of the survey along with the predictions of the POLR model are shown in Fig. 1.

Qualitatively, the data in Fig. 1 show that students' responses are not binary but show a gradually decreasing trend with taxonomic distance (i.e., a decreasing fraction of high-level responses and an increasing fraction of low-level responses). POLR analysis shows significant effects of all three predictor variables. First, students are more likely to agree that more closely related organisms have a common ancestor. This was also found among the students who gave a full-credit definition of common ancestor (data not shown). This is consistent with Poling and Evans' (2004) results and the results of the pilot study. Second, when questions included humans, students were less likely to agree that the pair had a

Table 5 Results of POLR analysis of students' responses to versions $\mathrm{C}$ and $\mathrm{D}$

\begin{tabular}{lll}
\hline Factor & Coefficient & $P$ value \\
\hline $\begin{array}{lll}\text { One level increase } \\
\text { in taxonomic difference }\end{array}$ & -0.61 & $<10^{-16}$ \\
Humans included in question & -0.21 & 0.020 \\
Defined common ancestor & -0.25 & 0.0006
\end{tabular}

The coefficient is the natural logarithm of the change in the odds ratio resulting from a change in the corresponding factor 


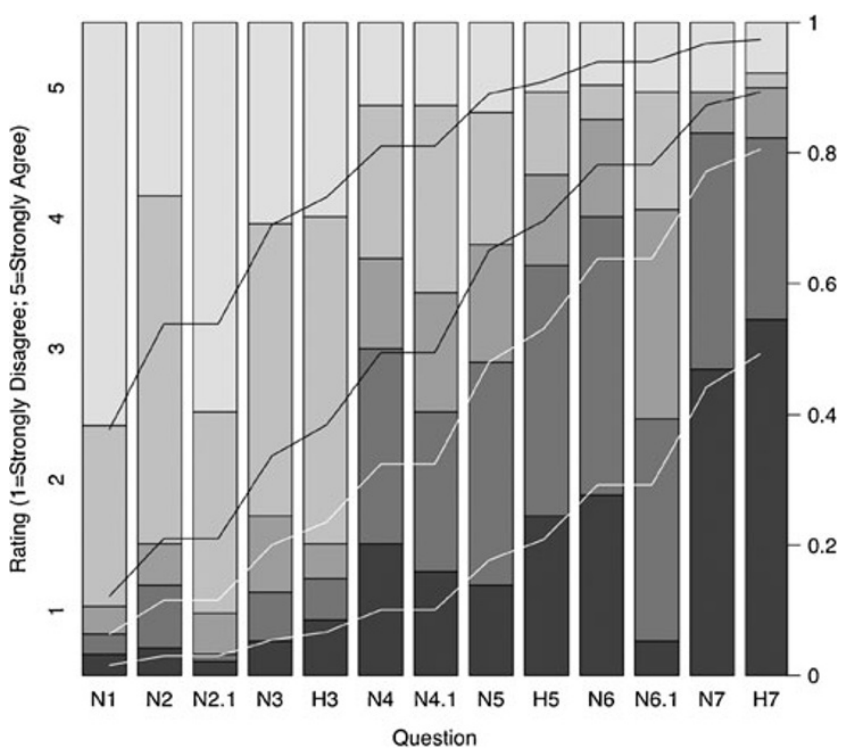

Fig. 1 Students' responses to version C of the survey. Question designations refer to the designations in Table 2. The vertical bars indicate the fraction of student responses at each level for each question: level 1 ("Strongly Disagree") at the bottom; level 5 ("Strongly Agree") at the top. The stepped diagonal lines indicate the predicted breakpoints between each of the levels for each question produced by the POLR model

common ancestor than with questions that did not include humans. Finally, and surprisingly, students who were asked to define common ancestor before responding to the statements (version C) were less likely to agree that pairs of organisms had a common ancestor than those not asked to define common ancestry before responding (version $\mathrm{D}$ ). The relative magnitudes of the different coefficients shows that taxonomic distance had the greatest effect on students' responses.

One exception to the negative correlation between students' response and taxonomic distance is question 10 (N6.1), which asked if earthworms and mosquitoes had a common ancestor. In general, students were more likely to think that these had a common ancestor than the taxonomic distance between the two would predict. One possible explanation is that students are unaware of the degree of diversity between different invertebrate phyla and lump all invertebrates into one large group of "nonvertebrates."

We next determined if the negative correlation between response and taxonomic distance observed in the class as a whole was observable at the level of individual students. For each student, we calculated the Kendall's tau correlation coefficient between their responses and the taxonomic distance of each statement. A box plot of these data is shown in Fig. 2. A similar distribution of tau values was also found for students who gave a full-credit definition of common ancestor. These data show that the overwhelming majority of students, even those who could correctly define "common ancestor," individually show a negative correlation between level of agreement and taxonomic distance.

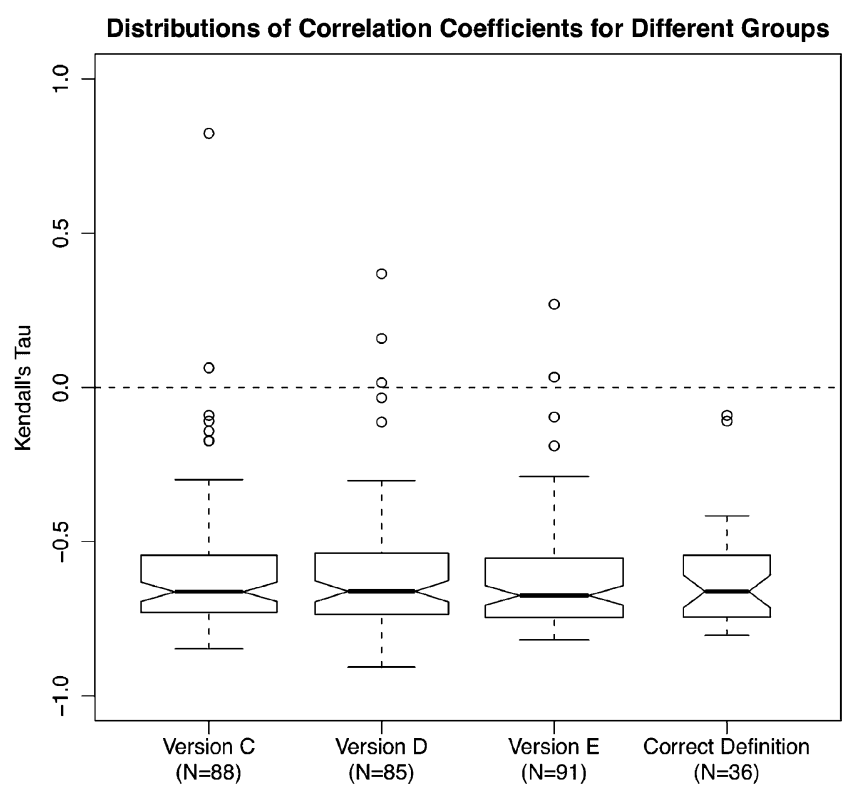

Fig. 2 Distributions of correlation coefficients for different groups. Box plots of the correlation coefficients between taxonomic distance and each student's responses are displayed above. The heavy line in the box indicates the median; the box itself includes the first and third quartiles; the whiskers extend to the most extreme point that is more than $1.5 \times$ the interquartile range; the circles indicate outliers. The versions above correspond to the survey versions in Table 2; "Correct Definition" corresponds to the students who completed version $\mathrm{C}$ of the survey and correctly defined common ancestry

The preceding analyses showed that students' response levels were affected by several factors, the strongest of which was taxonomic distance. To determine if there was any structure to the students' responses to the questions, we performed a principal components analysis on the pooled data from students who had completed version $\mathrm{C}$ or $\mathrm{D}$. The results of this analysis are shown in Table 6. The first two principal components explain a majority $(61 \%)$ of the variance in student responses. Using a cutoff of 0.25 , the

Table 6 Results of principal components analysis of students' responses to versions $\mathrm{C}$ and $\mathrm{D}$

The question designations refer to the designations in Table 2. $\mathrm{PC} 1$ and $\mathrm{PC} 2$ refer to the first and second principal components, respectively. Loadings greater than 0.25 are highlighted in bold type

\begin{tabular}{lll}
\hline Question & PC1 & PC2 \\
\hline N1 & 0.105 & $\mathbf{- 0 . 5 3 3}$ \\
N2 & 0.182 & $-\mathbf{0 . 2 8 8}$ \\
N2.1 & 0.084 & $-\mathbf{0 . 4 9 2}$ \\
N3 & 0.169 & $-\mathbf{0 . 4 1 5}$ \\
H3 & 0.166 & $-\mathbf{0 . 2 8 2}$ \\
N4 & $\mathbf{0 . 3 1 6}$ & -0.009 \\
N4.1 & $\mathbf{0 . 3 2 1}$ & -0.065 \\
N5 & $\mathbf{0 . 2 8 8}$ & 0.050 \\
H5 & $\mathbf{0 . 3 4 5}$ & 0.201 \\
N6 & $\mathbf{0 . 3 6 7}$ & 0.184 \\
N6.1 & $\mathbf{0 . 3 1 0}$ & 0.078 \\
N7 & $\mathbf{0 . 3 5 7}$ & 0.154 \\
H7 & $\mathbf{0 . 3 5 9}$ & 0.167 \\
\hline
\end{tabular}


first two principal components correspond roughly to highdiversity pairs (difference levels 4-7) and low-diversity pairs (levels 1-3), respectively. Interestingly, these principal components are based on taxonomic distance alone, not on the presence or absence of humans in the question. This further supports our finding that students view taxonomic distance as the most salient factor in determining the likelihood of common ancestry.

The last set of analyses were carried out on the data from students who had completed version E of the survey- the version with a three-level (1=no, $2=$ don't know, $3=y e s)$ simplified Likert-type response scale. These data along with the POLR predicted lines are shown in Fig. 3. The POLR model predicted the response significantly better than a null model with no parameters (model likelihood $\chi^{2}<0.0001$ ), and it explained roughly $45 \%$ of the variance in responses (Nagelkerke's $R^{2}=0.45$ ). POLR analysis showed a significant effect of taxonomic distance (coefficient $=-0.88, p<10^{-16}$ ) but no significant effect of including humans in the pair $(p=0.15)$. Thus, the major finding with versions $\mathrm{C}$ and $\mathrm{D}$, the inverse relationship between taxonomic distance and agreement, was also found with a no/don't-know/yes response scale.

\section{Conclusions and Recommendations for Teaching}

Our data provide a snapshot of an important group of students, beginning biology undergraduates. These students have had K-12 education and one semester of college biology and will

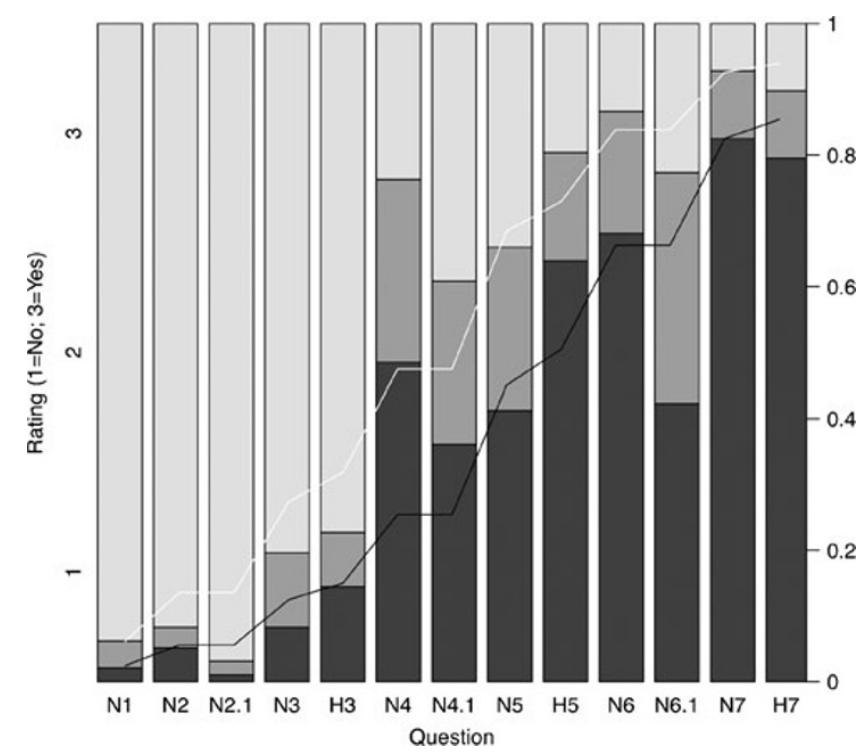

Fig. 3 Students' responses to version E of the survey. Question designations refer to the designations in Table 2 . The vertical bars indicate the fraction of student responses at each level for each question: level 1 ("No") at the bottom, level 2 ("Not Sure"), level 3 ("Yes") at the top. The stepped diagonal lines indicate the predicted breakpoints between each of the levels for each question produced by the POLR model typically move on to courses that emphasize evolution and go on to careers where an understanding of evolution is critical. Our data thus provide information for faculty who will teach students at this level as well as those who prepare students for this level. Our data show a consistent pattern with three main components; together, they have important implications in the classroom.

First, that, although a significant minority students can give a satisfactory definition of common ancestor, only a very small percentage can operationalize that definition properly when asked if particular pairs of organisms have a common ancestor. Fewer than $12 \%$ of the students chose "Strongly Agree" or "Yes" for all of the questions. The ability to define a concept without being able to apply it to particular cases is common in education and has been observed in other fields. For example, Pinarbasi and Canpolat (2003) found that while undergraduate chemistry students could correctly define terms related to solution chemistry (for example, unsaturated, saturated, and supersaturated solutions) on an open-response exam question, only $16.8 \%$ could correctly apply these definitions to an example. Our results reveal a particularly extreme case of a deeply held misconception. This suggests that instructors should pay particular attention to making sure that students can apply this idea instead of just being able to define it.

Although it is possible that students' incorrect responses could be due to the unusual nature of the survey response choices - answering what is essentially a "yes or no" question in five "shades of gray" and creating a situation where the correct answer is the same choice for all 13 questionsseveral of our findings make this explanation unlikely. First, the students' answers are not random - the students are not guessing as a response to the unusual nature of the survey. Instead, their responses show a consistent correlation between level and taxonomic distance even when the taxonomic order of the statements is scrambled. Second, when asked the same questions with no/don't-know/yes response choices (version E), students response levels still showed a strong and significant effect of taxonomic distance. Thus, it is likely that the students' responses genuinely reflect their inability to apply an understanding of common descent to these pairs of organisms.

Second, students' agreement levels show a strong negative correlation with increasing taxonomic distance in all versions of the survey. This is similar to the findings of Poling and Evans (2004) and echo Darwin's ambivalence quoted in the "Introduction" section. This is not surprising, given that superficial features of distantly related organisms can differ so greatly. In addition, students show "human exceptionalism" (Miller et al. 2006) - the idea that humans stand apart from other organisms - when they give lower agreement levels to statements including humans. It is surprising that students still hold these ideas after K-12 education that discusses 
common ancestry and a semester of cell and molecular biology that emphasizes the unity of life at the molecular level. Again, this is similar to findings in many other disciplines that many misconceptions are quite resistant to change (Tanner and Allen 2005).

Finally, our results show that addressing this misconception - that common ancestry is a continuous property that varies with taxonomic difference-in a specific context is likely to be more successful than simply giving a definition of common ancestry. This is shown by comparing the effect of question order (version A vs. version B) with the effect of asking for a definition of common ancestry (version C vs. version D). Our results show that exposing students to high-diversity organism sets raised their confidence in common ancestry, while asking for a definition of common ancestry reduced their confidence. This suggests that a successful approach would involve raising this misconception early in the course, perhaps by discussing Darwin's difficulties and relating them to the students' responses. Potentially, students could complete the survey, either on paper or as a clicker quiz, and then the instructor could discuss the results, pointing out how few students got it correct. This could be followed with a discussion emphasizing the connections between any pair of living things, perhaps with specific examples of the common ancestors of different pairs of organisms. These measures are only a start; it is clear from experience with other misconceptions that the issue must be raised repeatedly in multiple contexts if students are to learn the correct idea. Fortunately, common ancestry is a concept that recurs throughout courses on evolution and diversity.

In future studies, it will be important to see the extent to which our findings apply in other cases. Poling and Evans
(2004) showed that religiosity was negatively correlated with understanding many facets of evolution, including common ancestry. It would be interesting to see how students from regions with stronger creationist movements perform on this survey. In addition, it would be interesting to give the survey to upper-level biology students to see the effects of upperlevel courses on the prevalence of this misconception.

\section{References}

Darwin CR. The origin of the species. New York: Random House; 1859. Freeman S, Herron JC. Evolutionary analysis. Upper Saddle River, NJ: Prentice Hall; 2007.

MassDOE. Massachusetts Science and Technology/Engineering Curriculum Framework. 2006. www.doe.mass.edu/frameworks/scitech/ 1006.pdf. Accessed 25 Feb 2011.

Mildenhall P, Williams J. Instability in students' use of intuitive and Newtonian models to predict motion: the critical effect of the parameters involved. Int J Sci Educ. 2001;23(6):643-60.

Milller JD, Scott EC, Okamoto S. Public acceptance of evolution. Science. 2006;313(5788):765.

Nadelson LS, Southerland SA. Development and preliminary evaluation of the measure of understanding of macroevolution: introducing the MUM. J Exp Educ. 2010;78(2):151-90.

NRC. National Science Education Standards (1996). Washington, DC.: National Academy Press.

Pinarbasi T, Canpolat N. Students' understanding of solution chemistry concepts. J Chem Educ. 2003;80(11):1328-32.

Poling D, Evans M. Religious belief, scientific expertise, and folk ecology. J Cogn Cult: Stud Cogn Anthropol Sci. 2004;4(3):485-524.

Swanson CB. Evolution in state science education standards. Editorial Projects in Education Research Center Volume. 2005

Tanner K, Allen D. Approaches to biology teaching and learning: understanding the wrong answers - teaching toward conceptual change. CBE-Life Sci Educ. 2005;4:112-7.

Venables WN, Ripley BD. Modern applied statistics with s. New York: Springer; 2002. 